Journal of Applied Fluid Mechanics, Vol. 4, No. 4, pp. 85-89, 2011.

Available online at www.jafmonline.net, ISSN 1735-3572, EISSN 1735-3645.

DOI: $10.36884 / \mathrm{jafm} .4 .04 .11950$

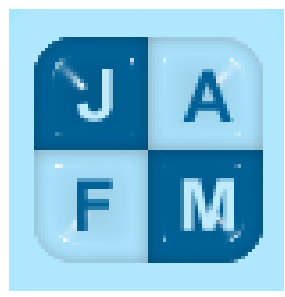

\title{
On the Flow of a Paramagnetic Fluid in a Differentially Heated Channel
}

\author{
H. Sadat ${ }^{\dagger}$ and C. Prax \\ Institut PPRIME, Université de Poitiers, 40 Avenue du Recteur Pineau, Poitiers, France \\ †Corresponding Author Email: hamou.sadat@univ-poitiers.fr
}

(Received October 9, 2009; accepted June 13, 2010)

\begin{abstract}
In the present study, we investigate the flow of a paramagnetic fluid in a two dimensional heated channel when an external magnetic gradient is imposed. In the fully developed regime, an analytical solution shows that a flow reversal may occur; the condition of this is given $n$ terms of the Reynolds number. Numerical simulations are then carried out for more general situations. It is shown that the analytical model gives good qualitative predictions.
\end{abstract}

Keywords: Paramagnetic fluid, Flow reversal, Channel flow, Magnetohydrodynamics.

\section{INTRODUCTION}

The ability to control flow and heat transfer in electrically conductive fluids has long been recognized (Amaouche et al. 2005; Amaouche et al. 2007; Ghosh 2001). In the case of paramagnetic non conducting fluids where the Lorentz force is useless, it has been shown that Kelvin forces can be used efficiently in many situations (Yang et al. 2003; Akamatsu et al. 2003; Khaldi et al. 2005; Sophy et al. 2005; Khaldi et al. 2001; Uetake et al. 2000). Further, the variation of the magnetic susceptibility (and thus of Kelvin forces) with temperature according to Curie-Weiss law (in the case of paramagnetic fluids), allows the possibility to generate and control the fluid motion. One of the first experimental evidence of this phenomenon has been made in 1991 (Braithwaite et al. 1991). Since then, many studies have been done as the applications are numerous in many fields. Recent advances in material science have allowed for the commercial manufacture of rare earth permanent magnets with strengths on the order of or even greater than 1 Tesla. This allows the possibility of i) generating magnetoconvection with a sufficient effect in microgravity environment or ii) control of natural and forced convection. When the considered fluids have low or small conductivity, the magnetic Reynolds and Prandtl numbers are small and the induced magnetic fields are negligible compared to the exterior magnetic field. The additional body force can then be deduced from the concept of free energy of Helmholtz (Landau et al. 1960) and can be written (in the presence of a gradient of magnetic field) in the form:

$$
\vec{F}=\frac{\chi(T)}{\mu_{0}} \vec{B} \cdot \vec{\nabla} \vec{B}
$$

Where $\chi$ and $\mu_{0}$ are respectively the magnetic susceptibility and permeability of vacuum which are related to the medium permeability $\mu$ by the following relations: $\mu_{r}-1=\chi$ and $\mu_{r}=\frac{\mu}{\mu_{0}}$.

The main goal of this paper is to show that a magnetic field can cause the reversal of flow in a channel. For one, an analytical model is developed in the case of a fully developed flow leading to the definition of a reversal condition. Secondly, numerical results are presented for more general and realistic situations where the reversal phenomenon is highlighted.

\section{Problem Statement}

Consider the flow of a paramagnetic fluid in a channel sketch in Fig. 1. Suppose that the walls are maintained to temperature $T_{1}$ and $T_{2}$ with $T_{1}>T_{2}$. A magnet device provides a non uniform magnetic field shown in Fig. 2. Temperature gradient in the fluid leads to a spatial inhomogeneity of the Kelvin force (Eq.1). This force is always headed in the direction of higher magnetic fields, but is more intense on fresh air near the cold wall.

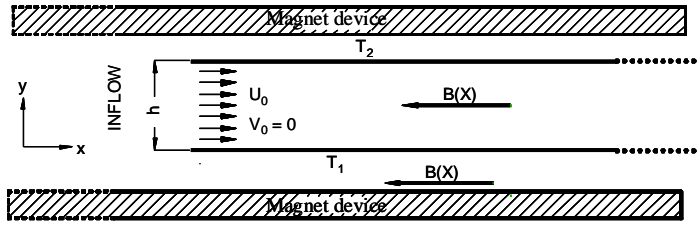

Fig. 1. Schematic configuration of the channel problem in presence of magnetic gradient field 


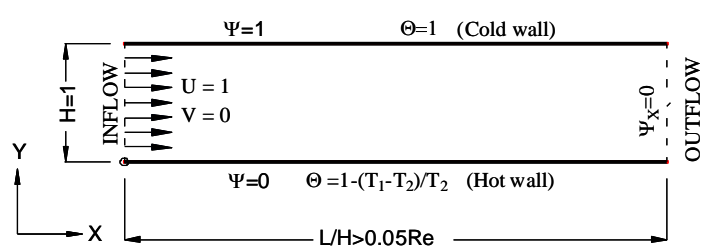

Fig. 2. Calculation domain with boundary conditions and truncate rule

\section{FULLY DEVELOPED ANALYTICAL SOLUTION}

If we suppose the existence of a fully developed flow, the governing equations of the problem are:

$\frac{1}{\rho} \frac{d P}{d x}=v \frac{d^{2} u}{d y^{2}}+\frac{A}{T} B \frac{d B}{d x}$

$u \frac{\partial T}{\partial x}=\alpha \frac{\partial^{2} T}{\partial y^{2}}$

Assume now that $\mathrm{T}=\mathrm{Tr}+\theta$ where $\operatorname{Tr}$ is a given temperature of reference. At first order one can writes:

$\frac{1}{T} \cong \frac{1}{T_{r}}\left(1-\frac{\theta}{T_{r}}\right)$

By using the following variable changes: $X=\frac{x}{h}$; $Y=\frac{y}{h} ; \quad P^{*}=\frac{h^{2}}{\rho v^{2}} p ; \quad U=\frac{u}{U_{0}} ; \Theta=1-\frac{\theta}{T_{r}}$, and by introducing the Reynolds number $\operatorname{Re}=\frac{U_{0} h}{v}$, and the Prandtl number $\operatorname{Pr}=\alpha / v$, the Eqs. (3) and (4) become:

$\frac{d P^{*}}{d X}=\frac{1}{\operatorname{Re}} \frac{d^{2} U}{d Y^{2}}+\frac{R}{\operatorname{Re}^{2}} \Theta$

$\operatorname{Re} \operatorname{Pr} U \frac{\partial \Theta}{\partial X}=\frac{\partial^{2} \Theta}{\partial Y^{2}}$

where: $R=\frac{h^{2}}{v^{2}} \frac{\chi_{0} T_{0}}{\mu_{0} T_{r}} B \frac{d B}{d X}$

In fully developed flow, $\frac{d P^{*}}{d X}$ is constant and $\Theta$ is a function of $Y$ only (see Annex). If we suppose that the fluid is flowing in the positive direction and that $\mathrm{R}$ is constant (as it is the case in experimental conditions of Braithwaite et al. (1991), for example) and that $\mathrm{T}_{\mathrm{r}}=\mathrm{T}_{2}$, it can be shown that the analytical solutions are:

$\Theta=(1-r) Y+r$

$\frac{U}{\operatorname{Re}}=\left(\frac{d P^{*}}{d X}-\frac{R}{\operatorname{Re}^{2}} r\right)\left(\frac{Y^{2}-Y}{2}\right)$

$-\frac{R}{\mathrm{Re}^{2}}(1-r)\left(\frac{Y^{3}-Y}{6}\right)$

where: $\quad r=\frac{2 T_{2}-T_{1}}{T_{2}}$

The previous form of adimensional speed clearly shows that situations may exist where the flow is reversed. They correspond to cases where:
$U^{\prime}(0) U^{\prime}(1)>0$

This condition leads to:

$\frac{R(r+2)}{3 \operatorname{Re}^{2}}<\frac{d P^{*}}{d X}<\frac{R(1+2 r)}{3 \operatorname{Re}^{2}}$

Expressed in terms of Reynolds number, the previous condition leads to the following inequalities (according to the sign of R):

$\begin{array}{ll}\operatorname{Re}<\frac{(r-1)}{72} R & (\text { if } \mathrm{R}<0) \\ \operatorname{Re}<\frac{(1-r)}{72} R \quad(\text { if } \mathrm{R}>0)\end{array}$

Reintroducing to the physical variables (and by using the definition of R) these inequalities now read:

$U_{0}<\frac{(r-1) h}{72 v} \frac{\chi_{0} T_{0}}{\mu_{0} T_{r}} B \frac{d B}{d X} \quad($ if $\mathrm{R}<0)$
$U_{0}<\frac{(1-r) h}{72 v} \frac{\chi_{0} T_{0}}{\mu_{0} T_{r}} B \frac{d B}{d X} \quad($ if $\mathrm{R}>0)$

The flow reversal is therefore possible when the imposed inlet velocity is smaller than a given quantity whatever the sign of R: i) For a negative value of R, the magnetic forces are opposite to the flow direction and they act with more strength on cold fluid (because of the higher susceptibility of cold air). A reversal of the flow can occur when these magnetic forces are strong enough to produce an opposite motion which begins in the boundary layer where the velocity of fresh air is small ii) Even if not so intuitively evident, the model shows that the flow reversal is also possible for positive values of $\mathrm{R}$ while obviously the magnetic force is headed in the direction of the flow.

Equation (12) shows that in this case the pressure gradient becomes positive while at the same time the flow is still in the positive direction. The reason of this seems more subtle: Kelvin forces act to accelerate the flow but this acceleration is not uniform (stronger for fresh air, smaller for hot air); when these forces themselves are more than sufficiently strong to assume the generation of a flow as important as the imposed flow itself, by virtue of the mass conservation principle, a flow reversal takes place in the hot side.

The strong acceleration of fresh air (relatively to hot air acceleration) can lead to deform enough the velocity profile so that the net positive flow is entirely monitored by the cold side of the duct.

In order to get more insight on this phenomenon, this analytical model is confronted to two dimensional numerical experiments in the following sections.

\section{NUMERICAL VALIDATION}

Eliminating the pressure terms, the two-dimensional Navier-Stokes (where the external magnetic force is introduced) and energy equations can be written as follows:

$\Delta \Psi=-\Omega$ 
$U \cdot \frac{\partial \Omega}{\partial X}+V \cdot \frac{\partial \Omega}{\partial Y}=\frac{1}{\operatorname{Re}} \cdot \Delta \Omega-\frac{h^{2}}{\operatorname{Re}^{2} v^{2}} \cdot \frac{\chi_{293.15} \cdot 293.15}{\mu_{o} T_{2}}$

$\left(B(X) \cdot \frac{\partial B(X)}{\partial X} \cdot \frac{\partial \Theta}{\partial Y}+B(Y) \frac{\partial B(Y)}{\partial Y} \frac{\partial \Theta}{\partial X}\right)$

$U \cdot \frac{\partial \Theta}{\partial X}+V \cdot \frac{\partial \Theta}{\partial Y}=\frac{1}{\operatorname{RePr}} \Delta \Theta$

Where $\Omega=\frac{\omega \cdot h}{U_{0}}$ and $\Psi=\frac{\psi}{h U_{0}}$ are the adimensional vorticity and streamfunction.

The calculation domain $\left(\mathrm{H}^{*} \mathrm{~L}\right)$ with $\mathrm{L} / \mathrm{H}=5$ has been discretized with regular grids and the equations (16-18) have been solved by using a finite difference method. As shown in Fig. 2, Dirichlet and Neuman boundary conditions are used for streamlines and temperature for several magnetic conditions. $\Omega$-production takes place at walls and Eq. (17) describes diffusion and convection of this vorticity in the interior flow region. Boundaryvalues of vorticity at walls are obtained by using the conventionally first-order accurate formula. It takes the following form at the bottom wall:

$\omega_{j=1}=2\left(\psi_{i, j=1}-\psi_{i, j=2}\right) / \Delta Y^{2}$, where $\mathrm{i}, \mathrm{j}$ are the classical indexes and $\Delta Y$, the vertical grid step. The simulations have been carried out with air as working fluid $\left(v=1.5610^{-5} \mathrm{~m}^{2} / \mathrm{s}, \operatorname{Pr}=0.71\right)$ and with a reference temperature $\mathrm{T}_{2}=\mathrm{T}_{0}=293 \mathrm{~K}$.

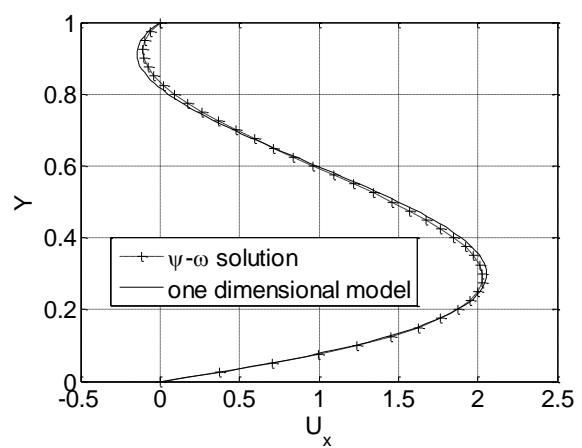

(a)

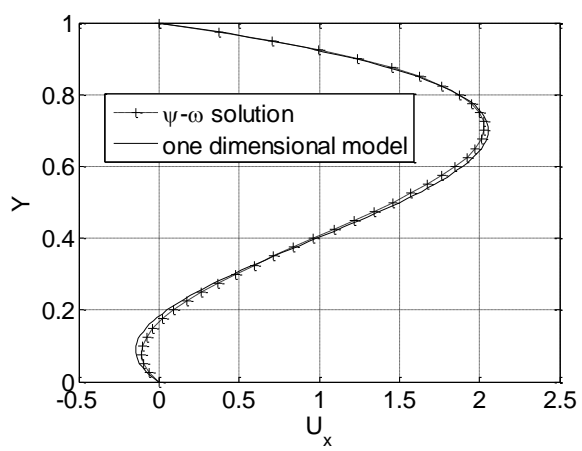

(b)

Fig. 3. Velocity profiles for $\mathrm{Re}=30$ and constant values of $\mathrm{R}$ : a) $\mathrm{R}=-2.10^{5}$ and b) $\mathrm{R}=2.10^{5}(\mathrm{r}=0.98294$, Condition (14) satisfied: $\operatorname{Re}=30<47.378$ )

\subsection{Uniform Magnetic Gradient (Constant Value of R)}

Let us suppose first that the magnetic gradient is constant, which is one of the assumptions used in the analytical model. Following Eq. (8), results are presented with negative and positive values of $R$ fixed to $\mathrm{R}= \pm 2.10^{5}$, and $\mathrm{R}= \pm 1.10^{5}$ while the temperature difference is $\left(\mathrm{T}_{1}-\mathrm{T}_{2}\right)=5 \mathrm{~K}$ leading to $\mathrm{r}=0.98294$.

For $\mathrm{Re}=30, \mathrm{R}= \pm 2.10^{5}$, the inequalities (14) are satisfied $(\operatorname{Re}<47.378)$. The velocity profiles at the channel exit are presented on Fig. 3 where it can be seen that the numerical results match perfectly the analytical model and that the flow reversal is indeed obtained. This is confirmed by the streamlines shown on Fig. 4 where the formation of a recirculation bubble is evidenced.

For $\mathrm{Re}=30, \mathrm{R}= \pm 1.10^{5}$, the inequalities (14) are not satisfied ( $\operatorname{Re}>23.689$ ); the analytical model does not predict a reversal as it can be seen on the velocity profiles of Fig. 5. The streamlines presented on Fig. 6 show that the flow is just deflected in the channel.

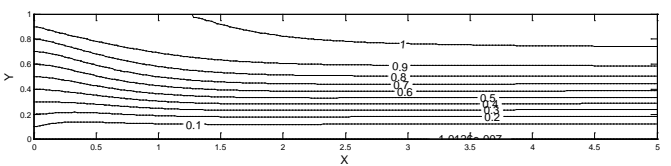

(a)

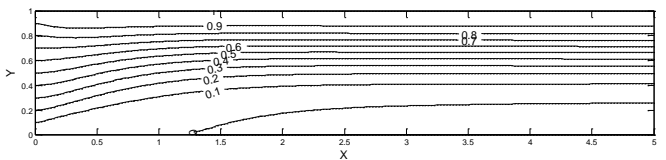

(b)

Fig. 4. Streamlines for $\mathrm{Re}=30$ and constant values of $\mathrm{R}$ : a) $R=-2.10^{5}$ and b) $R=2.10^{5}(r=0.98294$, Condition (14) satisfied: $\operatorname{Re}=30<47.378)$

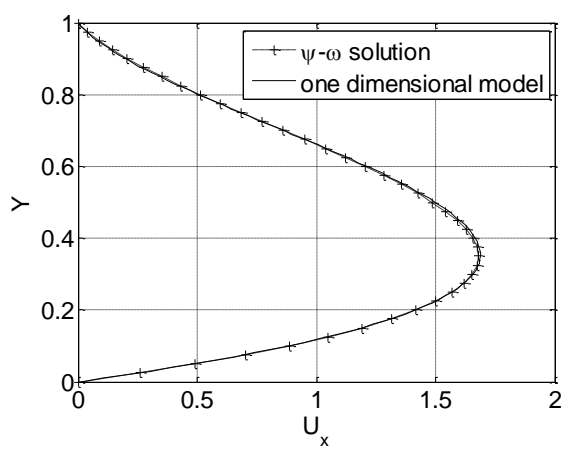

(a)

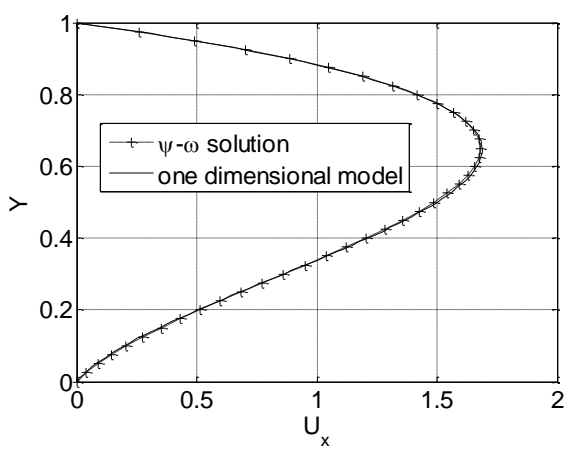

(b)

Fig. 5. Velocity profiles for $\mathrm{Re}=30$ and constant values of $\mathrm{R}$ : a) $\mathrm{R}=-1.10^{5}$ and $\left.\mathrm{b}\right) \mathrm{R}=1.10^{5}(\mathrm{r}=0.98294$, Condition (14) not satisfied : $\operatorname{Re}=30<23.689$ ) 


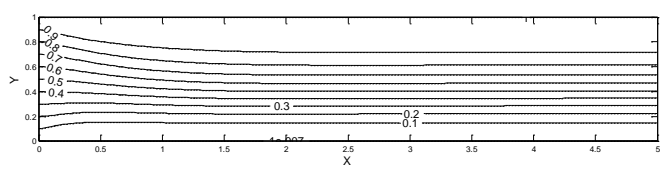

(a)

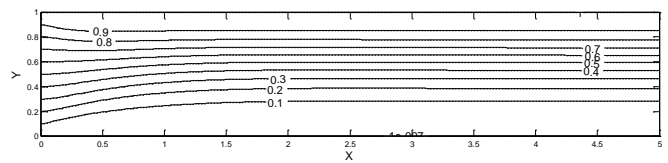

(b)

Fig. 6. Streamlines for $\mathrm{Re}=30$ and constant values of $\mathrm{R}$ : a) $\mathrm{R}=-1.10^{5}$ and b) $\mathrm{R}=1.10^{5}$ (r=0.98294, Condition (14) not satisfied : $\operatorname{Re}=30<23.689)$

\subsection{Channel in the Air Gap of Two Large Magnets Bar}

We now consider a more realistic situation where a portion of the channel is placed in the air gap of two magnet bars as shown on Fig. 7. The magnetic field is calculated according to the analytical solution of (Manikonda et al. 2006). We note $\mathrm{S}$ the adimensional dimension of the magnet faces and the distance between the two magnets is fixed to 1.02 . We also assume that rare earth permanent magnets of $1.25 \mathrm{~T}$. the axial line of magnetic device of is placed at the inflow channel, generating a magnetic field that decrease from the axial line to exterior of air gap, and from the vicinity of magnet faces to the axial line of the channel. The gradient of magnetic field is not uniform and is very wide in the vicinity of the corner of magnet bars $(X=3)$.

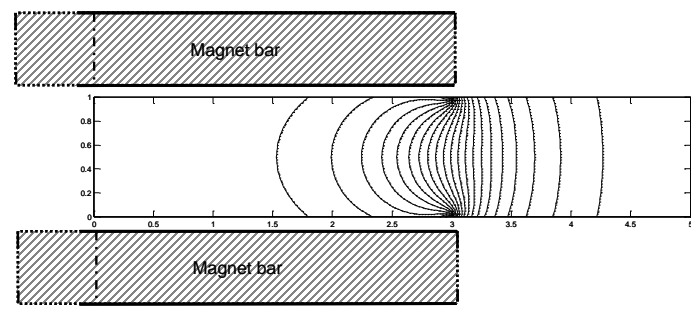

Fig. 7. Induction lines produced in the air-gap of magnet bars of adimensional inner section $S=6$, the magnetic axis is centred at $\mathrm{X}=0$

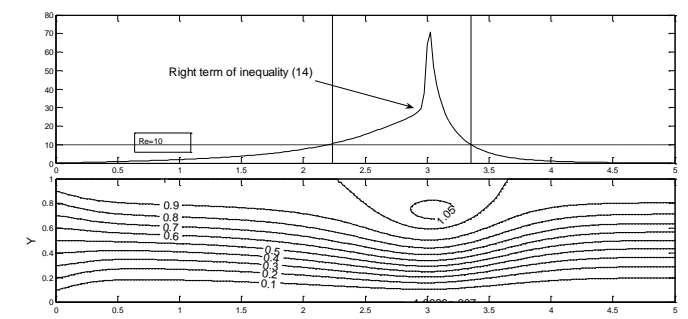

Fig. 8. Streamlines in the presence of magnet bars for $\mathrm{S}=6$ and estimation of inequality (14), $\mathrm{Re}=10$, $\mathrm{r}=0.98294$ Physical dimensions : $\mathrm{B}_{0}=1.25 \mathrm{~T}, \mathrm{~h}=1.5 \mathrm{~cm}$ $\mathrm{U}_{0}=1.04 \mathrm{~cm} / \mathrm{s},\left(\mathrm{T}_{1}-\mathrm{T}_{2}\right)=5 \mathrm{~K}$

The streamlines are presented on Fig. 8 for $\mathrm{Re}=10$ $\left(\mathrm{h}=1.5 \mathrm{~cm}, \quad \mathrm{U}_{0}=1.04 \mathrm{~cm} / \mathrm{s} \quad\right.$ and $\left.\quad\left(\mathrm{T}_{1}-\mathrm{T}_{2}\right)=5 \mathrm{~K}\right) ; \quad \mathrm{a}$ recirculation bubble in the vicinity of magnet corner near the cold wall (as predicted by the analytical model in this case) is developed. Even if the fully developed flow regime does not exist in the studied problem, we propose here to extend the scope of the analytical model by defining $R_{\text {mean }}(X)$, the average section value of the parameter $\mathrm{R}$ (Eq. (8)). This approach allows the evaluation of condition (14) as given in the upper part of Fig. 8. It can be seen that the satisfaction of the inequality (14) predicts accurately the beginning of the flow reversal, but underestimates the length of the bubble. It is worth noting that the central position of the bubble is located where the magnetic field gradient is maximal, at the exact level of exit of the air-gap.

To go further in the extension of the model, the velocity profile given by Eq. (10) is estimated by using the average value $R_{\text {avg, }}$, in the region where the inequality (14) is satisfied. It can be seen in Fig. 9, that the model is not too bad to qualitatively approach the profile the more marked by backflow effect, but in absence of fully developed regime, the effect is a little overestimated by the model.

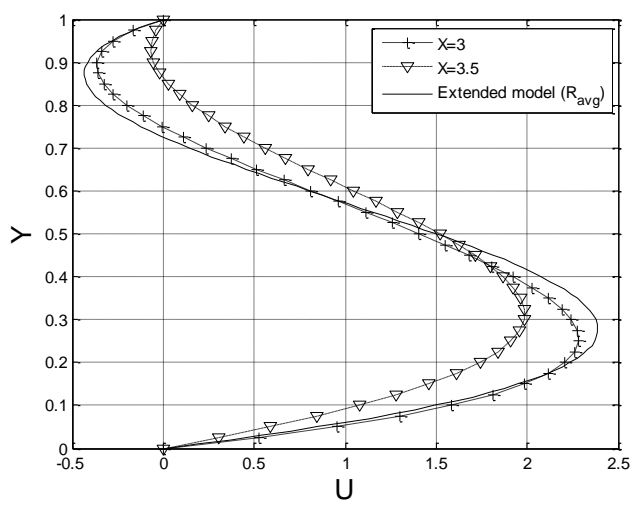

Fig. 9. Velocity profiles for $\mathrm{Re}=10, \mathrm{r}=0.98294$ and $\mathrm{R}$ avg $=-93501$ Physical dimensions : $\mathrm{B}_{0}=1.25 \mathrm{~T}, \mathrm{~h}=1.5 \mathrm{~cm}$ $\mathrm{U}_{0}=1.04 \mathrm{~cm} / \mathrm{s},\left(\mathrm{T}_{1}-\mathrm{T}_{2}\right)=5 \mathrm{~K}$

We now turn to examine a situation where the magnet axis is placed after the inflow section namely at $\mathrm{X}=5$ in Fig. 10; the magnetic field gradient is positive when $X<5$ and negative when $X>5$. The two predictable recirculation bubbles are once again obtained by simulations when the strength of Kelvin forces is strong enough. As shown on Fig. 11, they occur in the vicinity of the inlet section (along the hot wall) and of the outlet of the magnet air-gap (along the cold wall). As before, the satisfaction of the inequality (14) predicts accurately the beginning of the reversal, but underestimates the size of the bubbles. The central position of the cold bubble seems to be located at the exact level of exit of the air-gap as it was observed in the precedent simulation.

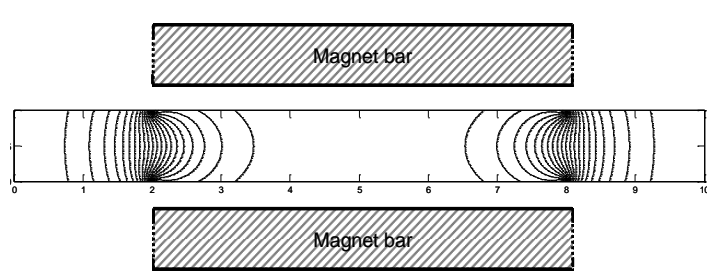

Fig. 10. Induction lines produced in the air-gap of magnet bars of adimensional inner section $S=6$, the magnetic axis is centred at $\mathrm{X}=5$ 
In turn, the central position of the hot bubble is not located where the magnetic field gradient is maximal and is delayed downstream the inlet of the air gap. An argument that can explain this can be advanced by looking the evolution of the right member of inequality (14) (given in the upper part of Fig. 11). The spatial evolution of this term is very stiff at the inlet of the magnet device while it evolves more progressively when the flow approaches the exit of air gap. Velocity profiles for the inlet and outlet sections of magnetic airgap are presented on Fig. 12 together with those given by the extended analytical model. A good agreement with the model is observed at the outlet $(X=8)$. The inlet profile (at $X=2$ ) does not match so well because of the delayed formation of the recirculation bubble.

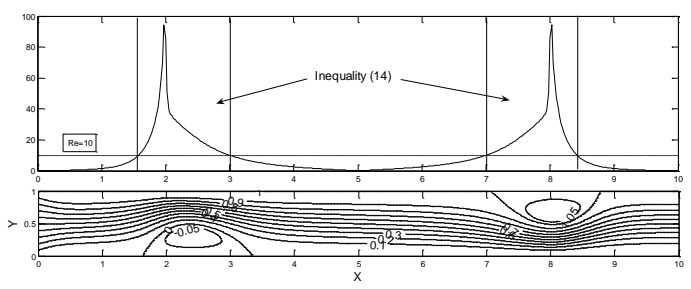

Fig. 11. Streamlines in the presence of magnet bars of adimensional inner section $S=6$, centred at $X=5$ and estimation of inequality (14), $\mathrm{Re}=10, \mathrm{r}=0.94883$

Physical dimensions: $\mathrm{B}_{0}=1.25 \mathrm{~T}, \mathrm{~h}=1 \mathrm{~cm}, \mathrm{U}_{0}=1.56 \mathrm{~cm} / \mathrm{s}$, $\left(\mathrm{T}_{1}-\mathrm{T}_{2}\right)=15 \mathrm{~K}$

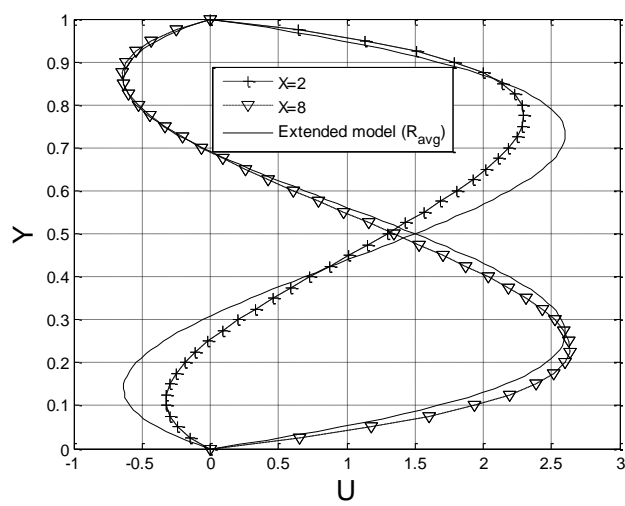

Fig. 12. Velocity profiles for $\mathrm{Re}=10, \mathrm{r}=0.94883$ and $\mathrm{R}_{\mathrm{avg}}= \pm 36705$; Physical dimensions: $\mathrm{B}_{0}=1.25 \mathrm{~T}, \mathrm{~h}=1 \mathrm{~cm}$, $\mathrm{U}_{0}=1.56 \mathrm{~cm} / \mathrm{s},\left(\mathrm{T}_{1}-\mathrm{T}_{2}\right)=15 \mathrm{~K}$

\section{CONCLuSion}

It was shown that an external magnetic field could lead to the partial reversal of the airflow in a twodimensional channel. A simplified model has been used to highlight the condition of inversion confirmed by numerical simulations. Although present results are obtained from simplified analytical and numerical models, we believe that the present study will shed the light on the behaviour of flows of paramagnetic fluids. Applications can be found in microfluidics and in low gravity environments.

\section{REFERENCES}

Amaouche, M., F. Nait Bouda and H. Sadat (2005).The onset of thermal instability of a two-dimensional hydromagnetic stagnation point flow. International Journal of Heat and MassTransfer 48 (21-22), 4435-4445.

Amaouche, M., F. Naït-Bouda and H. Sadat (2007). Oblique axisymmetric stagnation flows in magnetohydrodynamics. Phyics of Fluids 19, 114106.

Akamatsu, M., Higano, M., Takahashi, Y., Ozoe and H. (2003). Numerical computation on the control of aerial flow by the magnetizing force in gravitational and non-gravitational fields. Numerical Heat Transfer, Part A 43 (1), 9-29.

Braithwaite, D., E. Beaugnon and R.Tournier (1991). Magnetically controlled convection in a paramagnetic fluid. Nature 354, 134-136.

Ghosh, S.K. (2001). A note on unsteady hydromagnetic flow in a rotating channel permeated by an inclined magnetic field in the presence of an oscillator (Corrigendum and Addendum). Czechoslovak Journal of Physics 51(8), 799-804.

Khaldi, F., J. Noudem and P. Gillon (2005). On the similarity between gravity and magneto-gravity convection within a non-electroconducting fluid in a differentially heated rectangular cavity. International Journal of Heat and Mass Transfer 48(7), 1350-1360.

Khaldi, F. and P. Gillon (2001). Experimental and numerical investigation of thermomagnetic convection in a non electroconducting fluid. Comptes Rendus de l'Académie des Sciences, Mecanique 329(5), 357-362.

Landau, L.D. and E.M. Lifshitz (1960). Electrodynamics of Continuous Media. Pergamon Press, Oxford.

Manikonda, S. and M. Berz (2006). Multipole expansion solution of the Laplace equation using surface data. Nuclear Instruments and Methods in Physics Research, A 558.

Sophy, T., H. Sadat and L. Gbahoué (2005). Convection thermomagnétique dans une cavité différentiellement chauffée. International Communications in Heat and Mass Transfer 32(7) 923-930.

Uetake, H., N. Hirota, J. Nakagawa, Y. Ikezoe and K. Kitazawa (2000). Thermal convection control by gradient magnetic field. Journal of Applied Physics 87, 6310.

Yang, L., J. Ren, Y. Song and Z. Guo (2003). Free convection of a gas induced by a magnetic quadrupole field. Journal of Magnetism and Magnetic Materials 261(3), 377-384. 